Kragujevac Journal of Mathematics

Volume 45(4) (2021), Pages 623-633.

\title{
EXISTENCE OF POSITIVE SOLUTIONS FOR A PERTUBED FOURTH-ORDER EQUATION
}

\author{
MOHAMMAD REZA HEIDARI TAVANI ${ }^{1}$ AND ABDOLLAH NAZARI ${ }^{2}$
}

\begin{abstract}
In this paper, a special type of fourth-order differential equations with a perturbed nonlinear term and some boundary conditions is considered which is very important in mechanical engineering. Therefore, the existence of a non-trivial solution for such equations is very important. Our goal is to ensure at least three weak solutions for a class of perturbed fourth-order problems by applying certain conditions to the functions that are available in the differential equation (problem (1.1)). Our approach is based on variational methods and critical point theory. In fact, using a fundamental theorem that is attributed to Bonanno, we get some important results. Finally, for some results, an example is presented.
\end{abstract}

\section{INTRODUCTION}

In the present paper, the following fourth-order problem

$$
\left\{\begin{array}{l}
u^{(i v)}(x)=\lambda f(x, u(x))+h(u(x)), \quad x \in[0,1], \\
u(0)=u^{\prime}(0)=0, \\
u^{\prime \prime}(1)=0, \quad u^{\prime \prime \prime}(1)=\mu g(u(1)),
\end{array}\right.
$$

is studied, where $\lambda$ and $\mu$ are positive parameters, $f:[0,1] \times \mathbb{R} \rightarrow \mathbb{R}$ is non-negative $L^{1}$-Carathéodory function, $h: \mathbb{R} \rightarrow \mathbb{R}$ is a non-negative Lipschitz continuous function with the Lipschitz constant $0<L<1$, i.e.,

$$
\left|h\left(t_{1}\right)-h\left(t_{2}\right)\right| \leq L\left|t_{1}-t_{2}\right|
$$

for every $t_{1}, t_{2} \in \mathbb{R}$, and $h(0)=0$ and $g: \mathbb{R} \rightarrow \mathbb{R}$ is a non-positive continuous function. It is clear that for function $h$ we have $h(t) \leq L|t|$ for each $t \in \mathbb{R}$.

Key words and phrases. Fourth-order equation, weak solution, critical point theory, variational methods.

2010 Mathematics Subject Classification. Primary: 34B15. Secondary: 34B18, 58 E05.

DOI 10.46793/KgJMat2104.623H

Received: December 18, 2017.

Accepted: April 05, 2019. 
The problem (1.1) is related to the deflections of elastic beams based on nonlinear elastic. In relation with the problem (1.1), there is an interesting physical description.

An elastic beam of length $d=1$, which is clamped at its left side $x=0$, and resting on a kind of elastic bearing at its right side $x=1$ which is given by $\mu g$. Along its length, a load $\lambda f+h$, is added to cause deformations. If $u=u(x)$ denotes the configuration of the deformed beam, then since $u^{\prime \prime \prime}(1)$ represents the shear force at $x=1$, the condition $u^{\prime \prime \prime}(1)=\mu g(u(1))$ means that the vertical force is equal to $\mu g(u(1))$, which denotes a relation, possibly nonlinear, between the vertical force and the displacement $u(1)$.

Different models and their applications for problems such as (1.1) can be derived from [9]. Studying fourth-order differential equations are very important in engineering sciences. Therefore, several results are known concerning the existence of multiple solutions for fourth-order boundary value problems. For example, in [7] the author obtained the existence of at least two positive solutions for the problem

$$
\left\{\begin{array}{l}
u^{(i v)}(x)=f(x, u(x)), \quad x \in[0,1] \\
u(0)=u^{\prime}(0)=0, \\
u^{\prime \prime}(1)=0, \quad u^{\prime \prime \prime}(1)=g(u(1))
\end{array}\right.
$$

based on variational methods and maximum principle.

Moreover, in [8] authors considered iterative solutions for problem (1.2) with nonlinear boundary conditions. In particular, by using a variational methods the existence of non-zero solutions for problem (1.1) in the case of $h(t) \equiv 0$ has been established in [2]. In [6], using a critical points theorem obtained in [3], multiplicity results for the problem (1.1) were discussed. Also based on variational methods, existence and multiplicity results for this kind of problems were considered in $[4,5]$.

In the present paper, using a three critical points theorem obtained in [1] we will establish the existence of at least three weak solutions for the problem (1.1).

\section{PRELiminaries}

Our main tool is a three critical points theorem that we recall here in a appropriate form. This theorem has been established in [1]. In this theorem a suitable sign hypothesis is assumed.

Theorem 2.1. ([1, Corollary 3.1]). Let $X$ be a reflexive real Banach space, $\Phi: X \rightarrow$ $\mathbb{R}$ be a convex, coercive and continuously Gâteaux differentiable functional whose derivative admits a continuous inverse on $X^{*}, \Psi: X \rightarrow \mathbb{R}$ be a continuously Gâteaux differentiable functional whose derivative is compact, such that $\inf _{X} \Phi=\Phi(0)=$ $\Psi(0)=0$.

Assume that there are two positive constants $r_{1}, r_{2}$ and $w \in X$, with $2 r_{1}<\Phi(w)<$ $\frac{r_{2}}{2}$, such that

$$
\left(b_{1}\right) \frac{\sup _{u \in \Phi^{-1}(]-\infty, r_{1}[)} \Psi(u)}{r_{1}}<\frac{2}{3} \frac{\Psi(w)}{\Phi(w)} ;
$$


$\left(b_{2}\right) \frac{\sup _{u \in \Phi^{-1}(]-\infty, r_{2}[)} \Psi(u)}{r_{2}}<\frac{1}{3} \frac{\Psi(w)}{\Phi(w)}$

$\left(b_{3}\right)$ for each

$$
\lambda \in \Lambda_{r_{1}, r_{2}}:=\left(\frac{3}{2} \frac{\Phi(w)}{\Psi(w)}, \min \left\{\frac{r_{1}}{\sup _{u \in \Phi^{-1}(]-\infty, r_{1}[)} \Psi(u)}, \frac{\frac{r_{2}}{2}}{\sup _{u \in \Phi^{-1}(]-\infty, r_{2}[)} \Psi(u)}\right\}\right)
$$

and for every $u_{1}, u_{2} \in X$, which are local minimum for the functional $\Phi-\lambda \Psi$ and such that $\Psi\left(u_{1}\right) \geq 0$ and $\Psi\left(u_{2}\right) \geq 0$, one has

$$
\inf _{s \in[0,1]} \Psi\left(s u_{1}+(1-s) u_{2}\right) \geq 0 .
$$

Then, for each $\lambda \in \Lambda_{r_{1}, r_{2}}$ the functional $\Phi-\lambda \Psi$ has at least three distinct critical points which lie in $\Phi^{-1}(]-\infty, r_{2}[)$.

Now we give some preliminary definitions and basic concepts. Denote

$$
X:=\left\{u \in H^{2}[0,1] \mid u(0)=u^{\prime}(0)=0, u(1) \geq 0\right\},
$$

where $H^{2}[0,1]$ is the Sobolev space of all functions $u:[0,1] \rightarrow \mathbb{R}$ such that $u$ and its distributional derivative $u^{\prime}$ are absolutely continuous and $u^{\prime \prime}$ belongs to $L^{2}[0,1]$. Obviously, $X$ is a Hilbert space with the usual norm

$$
\|u\|_{X}=\left(\int_{0}^{1}\left(\left|u^{\prime \prime}(x)\right|^{2}+\left|u^{\prime}(x)\right|^{2}+|u(x)|^{2}\right) d x\right)^{1 / 2},
$$

which is equivalent to the norm

$$
\|u\|=\left(\int_{0}^{1}\left|u^{\prime \prime}(x)\right|^{2} d x\right)^{1 / 2} .
$$

The embedding $X \hookrightarrow C^{1}[0,1]$ is compact and also

$$
\|u\|_{C^{1}([0,1])}=\max \left\{\|u\|_{\infty},\left\|u^{\prime}\right\|_{\infty}\right\} \leq\|u\|,
$$

for each $u \in X$ (see [10]). We assume that the Lipschitz constant $L$ of the function $h$ satisfies $L<1$.

Definition 2.1. We mean by a (weak) solution of the problem (1.1), any function $u \in X$ such that

$$
\int_{0}^{1} u^{\prime \prime}(x) v^{\prime \prime}(x) d x-\lambda \int_{0}^{1} f(x, u(x)) v(x) d x+\mu g(u(1)) v(1)-\int_{0}^{1} h(u(x)) v(x) d x=0,
$$

holds for every $v \in X$.

Here, we note that if $f$ is continuous function, then every weak solution $u$ of the problem (1.1) is a classical solution (see [10, Lemma 2.1]).

Proposition 2.1. If $u_{0} \not \equiv 0$ is a weak solution for problem (1.1), then $u_{0}$ is nonnegative. 
Proof. Let $A=\left\{x \in[0,1] \mid u_{0}(x)<0\right\}$. Since $u_{0}$ is a weak solution for problem (1.1), then from (2.2) we have

$$
\begin{aligned}
& \int_{A \cup A^{c}} u_{0}^{\prime \prime}(x) v^{\prime \prime}(x) d x-\lambda \int_{A \cup A^{c}} f\left(x, u_{0}(x)\right) v(x) d x+\mu g\left(u_{0}(1)\right) v(1) \\
& -\int_{A \cup A^{c}} h\left(u_{0}(x)\right) v(x) d x=0,
\end{aligned}
$$

for every $v \in X$. Choosing $v(x)=\bar{u}_{0}=\max \left\{-u_{0}(x), 0\right\}$. Since $u_{0}$ is a weak solution for problem (1.1), then $u_{0}(1) \geq 0$ and hence $v(1)=0$. So, one has

$$
-\int_{A} v^{\prime \prime}(x) v^{\prime \prime}(x) d x+\lambda \int_{A} f\left(x, u_{0}(x)\right) u_{0}(x) d x+\int_{A} h\left(u_{0}(x)\right) u_{0}(x) d x=0,
$$

that is

$$
-\int_{A} v^{\prime \prime}(x) v^{\prime \prime}(x) d x=-\lambda \int_{A} f\left(x, u_{0}(x)\right) u_{0}(x) d x-\int_{A} h\left(u_{0}(x)\right) u_{0}(x) d x \geq 0,
$$

which means that $-\|v\|^{2} \geq 0$ and one has, $v=0$. Hence, $-u_{0} \leq 0$, that is, $u_{0} \geq 0$ and the proof is complete.

Put

$$
\begin{aligned}
F(x, t) & =\int_{0}^{t} f(x, \xi) d \xi, \quad \text { for all }(x, t) \in[0,1] \times \mathbb{R}, \\
G(t) & =\int_{0}^{t} g(\xi) d \xi, \quad \text { for all } t \in \mathbb{R}, \\
G_{\eta} & =\min _{|t| \leq \eta} G(t)=\inf _{|t| \leq \eta} G(t), \quad \text { for all } \eta>0,
\end{aligned}
$$

and

$$
H(t)=\int_{0}^{t} h(\xi) d \xi, \quad \text { for all } t \in \mathbb{R} .
$$

We state the following proposition which will be used in the next sections.

Proposition 2.2. ([6, Proposition 2.2]) Let $T: X \rightarrow X^{*}$ be the operator defined by

$$
T(u)(v)=\int_{0}^{1} u^{\prime \prime}(x) v^{\prime \prime}(x) d x-\int_{0}^{1} h(u(x)) v(x) d x,
$$

for each $u, v \in X$. Then $T$ admits a continuous inverse on $X^{*}$.

Now, we introduce the functional $I_{\lambda}: X \rightarrow \mathbb{R}$ associated with (1.1), $I_{\lambda}(u):=$ $\Phi(u)-\lambda \Psi(u)$ for all $u \in X$, where

$$
\Phi(u)=\frac{1}{2} \int_{0}^{1}\left|u^{\prime \prime}(x)\right|^{2} d x-\int_{0}^{1} H(u(x)) d x
$$

and

$$
\Psi(u)=\int_{0}^{1} F(x, u(x)) d x-\frac{\mu}{\lambda} G(u(1))
$$

for each $u \in X$. It is well known that $\Psi$ is a continuously Gâteaux differentiable functional whose differential at the point $u \in X$ is

$$
\Psi^{\prime}(u)(v)=\int_{0}^{1} f(x, u(x)) v(x) d x-\frac{\mu}{\lambda} g(u(1)) v(1)
$$


and furthermore, $\Psi^{\prime}: X \rightarrow X^{*}$ is a compact operator (see [10, page 1602]). Moreover, $\Phi$ is continuously Gâteaux differentiable functional whose differential at the point $u \in X$ is

$$
\Phi^{\prime}(u)(v)=\int_{0}^{1} u^{\prime \prime}(x) v^{\prime \prime}(x) d x-\int_{0}^{1} h(u(x)) v(x) d x,
$$

for every $v \in X$. Also according to Proposition 2.2, functional $\Phi$ whose derivative admits a continuous inverse on $X$ and moreover $\Phi$ is coercive and convex.

Definition 2.2. Let $\Phi$ and $\Psi$ be defined as above. Put $I_{\lambda}=\Phi-\lambda \Psi, \lambda>0$. We say that $u \in X$ is a critical point of $I_{\lambda}$ when $I_{\lambda}^{\prime}(u)=0_{\left\{X^{*}\right\}}$, that is, $I_{\lambda}^{\prime}(u)(v)=0$ for all $v \in X$.

Remark 2.1. We note that, the weak solutions of the problem (1.1) are exactly the critical points of the functional $I_{\lambda}$.

\section{MAin Results}

To get our result, fix three positive constants $\theta_{1}, \theta_{2}$ and $\delta$ such that

$$
\frac{12(1+L)\left(\frac{2}{3}\right)^{3} \pi^{4} \delta^{2}}{\int_{\frac{3}{4}}^{1} F(x, \delta) d x}<(1-L) \min \left\{\frac{\theta_{1}{ }^{2}}{\int_{0}^{1} \sup _{|t| \leq \theta_{1}} F(x, t) d x}, \frac{\theta_{2}{ }^{2}}{2 \int_{0}^{1} \sup _{|t| \leq \theta_{2}} F(x, t) d x}\right\}
$$

and take

$$
\lambda \in \Lambda:=\left[\frac{6(1+L)\left(\frac{2}{3}\right)^{3} \pi^{4} \delta^{2}}{\int_{\frac{3}{4}}^{1} F(x, \delta) d x}, \min \left\{\frac{(1-L) \theta_{1}{ }^{2}}{2 \int_{0}^{1} \sup _{|t| \leq \theta_{1}} F(x, t) d x}, \frac{(1-L) \theta_{2}{ }^{2}}{4 \int_{0}^{1} \sup _{|t| \leq \theta_{2}} F(x, t) d x}\right\}[\right.
$$

and set $\eta_{\lambda, g}$ given by

$$
\eta_{\lambda, g}:=\min \left\{\frac{2 \lambda \int_{0}^{1} \sup _{|t| \leq \theta_{1}} F(x, t) d x-(1-L) \theta_{1}^{2}}{2 G_{\theta_{1}}}, \frac{4 \lambda \int_{0}^{1} \sup _{|t| \leq \theta_{2}} F(x, t) d x-(1-L) \theta_{2}^{2}}{4 G_{\theta_{2}}}\right\},
$$

where $G_{\theta_{1}}$ and $G_{\theta_{2}}$ are assumed to be negative. It is easy to show that $\eta_{\lambda, g}>0$. Our main result is the following theorem.

Theorem 3.1. Suppose that there exist three positive constants $\theta_{1}, \theta_{2}$ and $\delta$, with $\frac{3}{4 \pi^{2}} \sqrt{\frac{3}{2}} \theta_{1}<\delta<\frac{3}{8 \pi^{2}} \sqrt{\frac{3(1-L)}{2(1+L)}} \theta_{2}$, such that

$$
\begin{aligned}
& \left(A_{1}\right) 12 \pi^{4}(1+L)\left(\frac{2}{3}\right)^{3} \delta^{2} \int_{0}^{1} \sup _{|t| \leq \theta_{1}} F(x, t) d x<(1-L) \theta_{1}^{2} \int_{\frac{3}{4}}^{1} F(x, \delta) d x ; \\
& \left(A_{2}\right) 24 \pi^{4}(1+L)\left(\frac{2}{3}\right)^{3} \delta^{2} \int_{0}^{1} \sup _{|t| \leq \theta_{2}} F(x, t) d x<(1-L) \theta_{2}^{2} \int_{\frac{3}{4}}^{1} F(x, \delta) d x .
\end{aligned}
$$


Then, for every $\lambda \in \Lambda$ and for each non-positive continuous function $g: \mathbb{R} \rightarrow \mathbb{R}$ there exists $\eta_{\lambda, g}>0$ given by (3.1) such that, for every $\left.\mu \in\right] 0, \eta_{\lambda, g}[$, the problem (1.1) admits at least three weak solutions $u_{i}$ for $i=1,2,3$, in $X$ such that $0 \leq u_{i}(x)<\theta_{2}$ for all $x \in[0,1], i=1,2,3$.

Proof. Our aim is to apply Theorem 2.1, to problem (1.1). For this purpose, fix $\lambda \in \Lambda$ and $\mu \in] 0, \eta_{\lambda, g}[$. Let $\Phi, \Psi: X \rightarrow \mathbb{R}$ be defined by

$$
\Phi(u)=\frac{1}{2} \int_{0}^{1}\left|u^{\prime \prime}(x)\right|^{2} d x-\int_{0}^{1} H(u(x)) d x
$$

and

$$
\Psi(u)=\int_{0}^{1} F(x, u(x)) d x-\frac{\mu}{\lambda} G(u(1)),
$$

for every $u \in X$. As seen before, the functionals $\Phi$ and $\Psi$ satisfy the regularity assumptions requested in Theorem 2.1. Put

$$
r_{1}:=\frac{(1-L)}{2} \theta_{1}^{2}, \quad r_{2}:=\frac{(1-L)}{2} \theta_{2}^{2}
$$

and

$$
w(x):= \begin{cases}0, & \text { if } x \in\left[0, \frac{3}{8}\right], \\ \delta \cos ^{2}\left(\frac{4 \pi x}{3}\right), & \text { if } \left.x \in] \frac{3}{8}, \frac{3}{4}\right], \\ \delta, & \text { if } x \in\left[\frac{3}{4}, 1\right] .\end{cases}
$$

We see that $w \in X$ and

$$
\|w\|^{2}=8 \pi^{4} \delta^{2}\left(\frac{2}{3}\right)^{3} .
$$

Now, according to (2.1), for every $u \in X$

$$
\frac{(1-L)}{2}\|u\|^{2} \leq \Phi(u) \leq \frac{(1+L)}{2}\|u\|^{2}
$$

holds and in particular

$$
4(1-L) \pi^{4} \delta^{2}\left(\frac{2}{3}\right)^{3} \leq \Phi(w) \leq 4(1+L) \pi^{4} \delta^{2}\left(\frac{2}{3}\right)^{3}
$$

Now, using $\frac{3}{4 \pi^{2}} \sqrt{\frac{3}{2}} \theta_{1}<\delta<\frac{3}{8 \pi^{2}} \sqrt{\frac{3(1-L)}{2(1+L)}} \theta_{2}$ and (3.4) we have $2 r_{1}<\Phi(w)<\frac{r_{2}}{2}$. Since, $\frac{(1-L)}{2}\|u\|^{2} \leq \Phi(u)$ for each $u \in X$ and for $i=1,2$, we see that

$$
\begin{aligned}
\left.\left.\Phi^{-1}(]-\infty, r_{i}\right]\right) & =\left\{u \in X \mid \Phi(u) \leq r_{i}\right\} \\
& \subseteq\left\{u \in X \mid \frac{(1-L)}{2}\|u\|^{2} \leq r_{i}\right\} \\
& \subseteq\left\{u \in X|| u(x) \mid \leq \theta_{i} \text { for each } x \in[0,1]\right\}
\end{aligned}
$$


and it follows that

$$
\begin{aligned}
\frac{\sup _{u \in \Phi^{-1}(]-\infty, r_{1}[)} \Psi(u)}{r_{1}} & =\frac{\sup _{u \in \Phi^{-1}(]-\infty, r_{1}[)}\left(\int_{0}^{1} F(x, u(x)) d x-\frac{\mu}{\lambda} G(u(1))\right)}{\frac{(1-L)}{2} \theta_{1}^{2}} \\
& \leq \frac{\int_{0}^{1} \sup _{|t| \leq \theta_{1}} F(x, t) d x-\frac{\mu}{\lambda} G_{\theta_{1}}}{\frac{(1-L)}{2} \theta_{1}^{2}} .
\end{aligned}
$$

On the other hand, since $w(x) \in[0, \delta]$ for each $x \in[0,1]$, we have

$$
\Psi(w)=\int_{0}^{1} F(x, w(x)) d x-\frac{\mu}{\lambda} G(w(1)) \geq \int_{\frac{3}{4}}^{1} F(x, \delta) d x-\frac{\mu}{\lambda} G(\delta) .
$$

Hence, we have

$$
\frac{\Psi(w)}{\Phi(w)} \geq \frac{\int_{\frac{3}{4}}^{1} F(x, \delta) d x-\frac{\mu}{\lambda} G(\delta)}{4(1+L) \pi^{4} \delta^{2}\left(\frac{2}{3}\right)^{3}} .
$$

Now, since $\mu<\eta_{\lambda, g}$ and $\lambda \in \Lambda$ one has

$$
\begin{aligned}
\frac{\int_{0}^{1} \sup _{|t| \leq \theta_{1}} F(x, t) d x-\frac{\mu}{\lambda} G_{\theta_{1}}}{\frac{(1-L)}{2} \theta_{1}^{2}} & \leq \frac{1}{\lambda}<\frac{\int_{\frac{3}{4}}^{1} F(x, \delta) d x}{6(1+L) \pi^{4} \delta^{2}\left(\frac{2}{3}\right)^{3}} \\
& \leq \frac{2}{3} \frac{\int_{\frac{3}{4}}^{1} F(x, \delta) d x-\frac{\mu}{\lambda} G(\delta)}{4(1+L) \pi^{4} \delta^{2}\left(\frac{2}{3}\right)^{3}} .
\end{aligned}
$$

So, from (3.5), (3.6) and (3.7), one has

$$
\frac{\sup _{u \in \Phi^{-1}(]-\infty, r_{1}[)} \Psi(u)}{r_{1}}<\frac{2}{3} \frac{\Psi(w)}{\Phi(w)}
$$

and hence, $\left(b_{1}\right)$ of Theorem 2.1 is established. As in the above process, we will have

$$
\begin{aligned}
\frac{2 \sup _{u \in \Phi^{-1}(]-\infty, r_{2}[)} \Psi(u)}{r_{2}} & \leq \frac{2\left(\int_{0}^{1} \sup _{|t| \leq \theta_{2}} F(x, t) d x-\frac{\mu}{\lambda} G_{\theta_{2}}\right)}{\frac{(1-L)}{2} \theta_{2}^{2}} \leq \frac{1}{\lambda}<\frac{\int_{\frac{3}{4}}^{1} F(x, \delta) d x}{6(1+L) \pi^{4} \delta^{2}\left(\frac{2}{3}\right)^{3}} \\
& \leq \frac{2}{3} \frac{\int_{\frac{3}{4}}^{1} F(x, \delta) d x-\frac{\mu}{\lambda} G(\delta)}{4(1+L) \pi^{4} \delta^{2}\left(\frac{2}{3}\right)^{3}} \leq \frac{2}{3} \frac{\Psi(w)}{\Phi(w)},
\end{aligned}
$$

that is,

$$
\frac{\sup _{u \in \Phi^{-1}(]-\infty, r_{2}[)} \Psi(u)}{r_{2}}<\frac{1}{3} \frac{\Psi(w)}{\Phi(w)}
$$

and hence, $\left(b_{2}\right)$ of Theorem 2.1 is established.

Finally, we will prove that $\Phi-\lambda \Psi$ satisfies the assumption $\left(b_{3}\right)$ of Theorem 2.1. Let $u_{1}$ and $u_{2}$ be two local minima for $\Phi-\lambda \Psi$. Then $u_{1}$ and $u_{2}$ are critical points 
for $\Phi-\lambda \Psi$, and so, they are weak solutions for the problem (1.1). According to Proposition 2.1 one has $u_{1}(x) \geq 0$ and $u_{2}(x) \geq 0$ for every $x \in[0,1]$. Hence, it follows that

$$
\inf _{s \in[0,1]} \Psi\left(s u_{1}+(1-s) u_{2}\right) \geq 0 .
$$

From Theorem 2.1, for every

$$
\left.\lambda \in \Lambda \subseteq \Lambda_{r_{1}, r_{2}}=\right] \frac{3}{2} \frac{\Phi(w)}{\Psi(w)}, \min \left\{\frac{r_{1}}{\sup _{u \in \Phi^{-1}(]-\infty, r_{1}[)} \Psi(u)}, \frac{r_{2} / 2}{\sup _{u \in \Phi^{-1}(]-\infty, r_{2}[)} \Psi(u)}\right\}[,
$$

the functional $\Phi-\lambda \Psi$ has at least three distinct critical points $u_{i}$, in $X$ such that $0 \leq u_{i}(x)<\theta_{2}$, for all $x \in[0,1], i=1,2,3$, which are the weak solutions of (1.1).

Remark 3.1. If in Theorem 3.1 we assume $f(x, 0) \neq 0$, then problem (1.1) has at least three distinct non-trivial and non-negative weak solutions.

Now, we present a variant of Theorem 3.1, which will be achieved by reversing the role of $\lambda$ and $\mu$.

Theorem 3.2. Suppose that there exist three positive constants $\theta_{1}, \theta_{2}$ and $\delta$, with $\frac{3}{4 \pi^{2}} \sqrt{\frac{3}{2}} \theta_{1}<\delta<\frac{3}{8 \pi^{2}} \sqrt{\frac{3(1-L)}{2(1+L)}} \theta_{2}$, such that

$$
\begin{aligned}
& \left(B_{1}\right) G(\delta)(1-L) \theta_{1}{ }^{2}<12 G_{\theta_{1}}(1+L)^{4} \delta^{2}\left(\frac{2}{3}\right)^{3} \\
& \left(B_{2}\right) G(\delta)(1-L) \theta_{2}{ }^{2}<24 G_{\theta_{2}}(1+L) \pi^{4} \delta^{2}\left(\frac{2}{3}\right)^{3} .
\end{aligned}
$$

Then, for each

$$
\left.\mu \in \Lambda^{\prime}:=\right] \frac{6(1+L) \pi^{4} \delta^{2}\left(\frac{2}{3}\right)^{3}}{-G(\delta)}, \min \left\{\frac{(1-L) \theta_{1}^{2}}{-2 G_{\theta_{1}}}, \frac{(1-L) \theta_{2}^{2}}{-4 G_{\theta_{2}}}\right\}[
$$

and for each non-negative $L^{1}$-Carathéodory function $f:[0,1] \times \mathbb{R} \rightarrow \mathbb{R}$ there exists $\eta_{\lambda, g}^{\prime}>0$, where

$$
\eta_{\lambda, g}^{\prime}=\min \left\{\frac{(1-L) \theta_{1}^{2}+2 \mu G_{\theta_{1}}}{2 \int_{0}^{1} \sup _{|t| \leq \theta_{1}} F(x, t) d x}, \frac{(1-L) \theta_{2}^{2}+4 \mu G_{\theta_{2}}}{4 \int_{0}^{1} \sup _{|t| \leq \theta_{2}} F(x, t) d x}\right\},
$$

such that, for all $\lambda \in] 0, \eta_{\lambda, g}^{\prime}[,(1.1)$ admits at least three weak solutions in $X$.

Proof. Fix $\mu \in \Lambda^{\prime}$ and $\left.\lambda \in\right] 0, \eta_{\lambda, g}^{\prime}[$. Let $\hat{\Psi}: X \rightarrow \mathbb{R}$ be defined by

$$
\hat{\Psi}(u)=\frac{\lambda}{\mu} \int_{0}^{1} F(x, u(x)) d x-G(u(1)),
$$


for each $u \in X$. We observe that $\Phi(u)-\lambda \Psi(u)=\Phi(u)-\mu \hat{\Psi}(u)$ for every $u \in X$. Choose $r_{1}, r_{2}$ and $w$ as given in (3.2) and (3.3). Now, we have

$$
\begin{aligned}
\frac{\sup _{u \in \Phi^{-1}(]-\infty, r_{1}[)} \hat{\Psi}(u)}{r_{1}} & =\frac{\frac{\lambda}{\mu} \int_{0}^{1} \sup _{|t| \leq \theta_{1}} F(x, t) d x-G_{\theta_{1}}}{\frac{(1-L)}{2} \theta_{1}^{2}} \leq \frac{1}{\mu}<\frac{-G(\delta)}{6(1+L) \pi^{4} \delta^{2}\left(\frac{2}{3}\right)^{3}} \\
& \leq \frac{2}{3} \frac{\frac{\lambda}{\mu} \int_{\frac{3}{4}}^{1} F(x, \delta) d x-G(\delta)}{4(1+L) \pi^{4} \delta^{2}\left(\frac{2}{3}\right)^{3}} \leq \frac{2}{3} \frac{\hat{\Psi}(w)}{\Phi(w)},
\end{aligned}
$$

that is,

$$
\frac{\sup _{u \in \Phi^{-1}\left(-\infty, r_{1}\right)} \hat{\Psi}(u)}{r_{1}}<\frac{2}{3} \frac{\hat{\Psi}(w)}{\Phi(w)}
$$

and

$$
\begin{aligned}
\frac{2 \sup _{u \in \Phi^{-1}(]-\infty, r_{2}[)} \hat{\Psi}(u)}{r_{2}} & =\frac{2\left(\frac{\lambda}{\mu} \int_{0}^{1} \sup _{|t| \leq \theta_{2}} F(x, t) d x-G_{\theta_{2}}\right)}{\frac{(1-L)}{2} \theta_{2}^{2}} \leq \frac{1}{\mu}<\frac{-G(\delta)}{6(1+L) \pi^{4} \delta^{2}\left(\frac{2}{3}\right)^{3}} \\
& \leq \frac{2}{3} \frac{\frac{\lambda}{\mu} \int_{\frac{3}{4}}^{1} F(x, \delta) d x-G(\delta)}{4(1+L) \pi^{4} \delta^{2}\left(\frac{2}{3}\right)^{3}} \leq \frac{2}{3} \frac{\hat{\Psi}(w)}{\Phi(w)},
\end{aligned}
$$

that is,

$$
\frac{\sup _{u \in \Phi^{-1}\left(-\infty, r_{2}\right)} \hat{\Psi}(u)}{r_{2}}<\frac{1}{3} \frac{\hat{\Psi}(w)}{\Phi(w)} .
$$

Therefore, since for each

$$
\left.\mu \in \Lambda^{\prime} \subseteq\right] \frac{3}{2} \frac{\Phi(w)}{\hat{\Psi}(w)}, \min \left\{\frac{r_{1}}{\sup _{u \in \Phi^{-1}(]-\infty, r_{1}[)} \hat{\Psi}(u)}, \frac{r_{2} / 2}{\sup _{u \in \Phi^{-1}(]-\infty, r_{2}[)} \hat{\Psi}(u)}\right\}[
$$

the assumptions of Theorem 2.1 are fulfilled, so the desired result is achieved from Theorem 2.1.

Now we will give a special case of Theorem 3.1 that the function $f$ depends only on $t$.

Corollary 3.1. Let $f: \mathbb{R} \rightarrow \mathbb{R}$ be a non-negative continuous function such that

$$
\lim _{t \rightarrow 0^{+}} \frac{f(t)}{t}=0
$$

and

$$
\int_{0}^{100} f(\xi) d \xi<\frac{625(1-L)}{6(1+L) \pi^{4}\left(\frac{2}{3}\right)^{3}} \int_{0}^{1} f(\xi) d \xi
$$


Also, suppose that, $1<\frac{300}{8 \pi^{2}} \sqrt{\frac{3(1-L)}{2(1+L)}}$. Then, for every

$$
\lambda \in] \frac{24(1+L) \pi^{4}\left(\frac{2}{3}\right)^{3}}{\int_{0}^{1} f(\xi) d \xi}, \frac{2500(1-L)}{\int_{0}^{100} f(\xi) d \xi}[
$$

and for every non-positive function $g: \mathbb{R} \rightarrow \mathbb{R}$ there exists $\delta_{\lambda, g}^{*}>0$ such that, for each $\mu \in\left[0, \delta_{\lambda, g}^{*}[\right.$, the problem

$$
\left\{\begin{array}{l}
u^{(i v)}(x)=\lambda f(u(x))+h(u(x)), \quad x \in[0,1] \\
u(0)=u^{\prime}(0)=0 \\
u^{\prime \prime}(1)=0, \quad u^{\prime \prime \prime}(1)=\mu g(u(1))
\end{array}\right.
$$

admits at least three classical solutions.

Proof. Our aim is to employ Theorem 3.1 by choosing $\theta_{2}=100$ and $\delta=1$. Hence, we have

$$
\frac{6(1+L)\left(\frac{2}{3}\right)^{3} \pi^{4} \delta^{2}}{\int_{\frac{3}{4}}^{1} F(x, \delta) d x}=\frac{24(1+L) \pi^{4}\left(\frac{2}{3}\right)^{3}}{\int_{0}^{1} f(\xi) d \xi}
$$

and

$$
\frac{(1-L) \theta_{2}{ }^{2}}{4 \int_{0}^{1} \sup _{|t| \leq \theta_{2}} F(x, t) d x}=\frac{2500(1-L)}{\int_{0}^{100} f(\xi) d \xi} .
$$

Also, according to the condition $1<\frac{300}{8 \pi^{2}} \sqrt{\frac{3(1-L)}{2(1+L)}}$, we have

$$
\delta<\frac{3}{8 \pi^{2}} \sqrt{\frac{3(1-L)}{2(1+L)}} \theta_{2} .
$$

Moreover, since $\lim _{t \rightarrow 0^{+}} \frac{f(t)}{t}=0$, one has

$$
\lim _{t \rightarrow 0^{+}} \frac{\int_{0}^{t} f(\xi) d \xi}{t^{2}}=0 .
$$

Then, there exists a positive constant $\theta_{1}<\frac{4 \pi^{2}}{3} \sqrt{\frac{2}{3}}$ such that

$$
\frac{\int_{0}^{\theta_{1}} f(\xi) d \xi}{\theta_{1}^{2}}<\frac{1-L}{48(1+L)\left(\frac{2}{3}\right)^{3} \pi^{4}} \int_{0}^{1} f(\xi) d \xi
$$

and

$$
\frac{\theta_{1}^{2}}{\int_{0}^{\theta_{1}} f(\xi) d \xi}>\frac{5000}{\int_{0}^{100} f(\xi) d \xi}
$$

Finally, a simple computation shows that all the circumstances of the Theorem 3.1 hold and so the desired result is achieved. 
Remark 3.2. If we consider

$$
f(t):= \begin{cases}18 t^{2}, & \text { if } t \leq 1 \\ -18000 t+18018, & \text { if } 1<t \leq 1.001 \\ 0, & \text { if } t>1.001\end{cases}
$$

and $h(t)=\frac{1}{2}|t|$ for all $t \in \mathbb{R}$, then we can consider $L=\frac{1}{2}$. In this case, a simple calculation reveals that, all the conditions of Corollary 3.1 are established.

Acknowledgements. The authors express their gratitude to the referees who reviewed this paper.

\section{REFERENCES}

[1] G. Bonanno and P. Candito, Non-differentiable functionals and applications to elliptic problems with discontinuous nonlinearities, J. Differential Equations 244 (2008), 3031-3059.

[2] G. Bonanno,A. Chinnì and S. Tersian, Existence results for a two point boundary value problem involving a fourth-order equation, Electron. J. Qual. Theory Differ. Equ. 33 (2015), 1-9.

[3] G. Bonanno and S. A. Marano, On the structure of the critical set of non-differentiable functions with a weak compactness condition, Appl. Anal. 89 (2010), 1-10.

[4] A. Cabada and S. Tersian, Multiplicity of solutions of a two point boundary value problem for a fourth-order equation, Appl. Math. Comput. 24 (2011), 1599-1603.

[5] M. R. Grossinho and St. A. Tersian, The dual variational principle and equilibria for a beam resting on a discontinuous nonlinear elastic foundation, Nonlinear Anal. 41(2000), 417-431.

[6] M. R. Heidari Tavani, Existence results for a perturbed fourth-order equation, J. Indones. Math. Soc. 23 (2017), 55-65.

[7] T. F. Ma, Positive solutions for a beam equation on a nonlinear elastic foundation, Math. Comput. Model. 39 (2004), 1195-1201.

[8] T. F. Ma and J. da Silva, Iterative solutions for a beam equation with nonlinear boundary conditions of third order, Appl. Math. Comput. 159 (2004), 11-18.

[9] S. Timoshenko, W. Weaver, Jr and D. H. Young, Vibrations Problems in Engineering, 5th Edition, John Wiley and Sons, New York, 1990.

[10] L. Yang, H. Chen and X. Yang, The multiplicity of solutions for fourth-order equations generated from a boundary condition, Appl. Math. Lett. 24 (2011), 1599-1603.

\footnotetext{
${ }^{1}$ Department of Mathematics,

RAMHORMOZ BRANCH,

ISLAMIC AZAD UNIVERSITY,

RAMHORMOZ, IRAN

Email address: m.reza.h56@gmail.com

${ }^{2}$ Department of Mathematics,

KAZERUN BRANCH,

ISLAMIC AZAD UNIVERSITY,

KAZERUN, IRAN

Email address: nazari_mat@yahoo.com
} 